\title{
Study of the Boson Peak and Fragility of Bioprotectant Glass-Forming Mixtures by Neutron Scattering
}

\author{
F. Migliardo, ${ }^{1}$ S. Magazù, ${ }^{1}$ M. A. Gonzalez, ${ }^{2}$ and C. Mondelli ${ }^{3}$ \\ ${ }^{1}$ Department of Physics and Earth Sciences, University of Messina, Viale D’Alcontres 31, P.O. Box 55, 98166 Messina, Italy \\ ${ }^{2}$ Institut Laue Langevin, 6 rue Jules Horowitz, 38042 Grenoble Cedex 9, France \\ ${ }^{3}$ CNR-IOM-OGG, Institut Laue Langevin, 6 rue Jules Horowitz, 38042 Grenoble Cedex 9, France
}

Correspondence should be addressed to F. Migliardo; fmigliardo@unime.it

Received 31 May 2013; Accepted 23 August 2013

Academic Editor: Gerhard Schumacher

Copyright (C) 2013 F. Migliardo et al. This is an open access article distributed under the Creative Commons Attribution License, which permits unrestricted use, distribution, and reproduction in any medium, provided the original work is properly cited.

\begin{abstract}
The biological relevance of trehalose, glycerol, and their mixtures in several anhydrobiotic and cryobiotic organisms has recently promoted both experimental and simulation studies. In addition, these systems are employed in different industrial fields, such as pharmaceutical and cosmetic industries, as additives in mixtures for cryopreservation and in several formulations. This review article shows an overview of Inelastic Neutron Scattering (INS) data, collected at different temperature values by the OSIRIS timeof-flight spectrometer at the ISIS Facility (Rutherford Appleton Laboratory, Oxford, UK) and by the IN4 and IN6 spectrometers at the Institut Laue Langevin (ILL, Grenoble, France), on trehalose/glycerol mixtures as a function of the glycerol content. The data analysis allows determining the Boson peak behavior and discussing the findings in terms of fragility in relation to the bioprotective action of trehalose and glycerol.
\end{abstract}

\section{Introduction}

In very high saline environments, halophile organisms tolerate extreme desiccation by passing into anhydrobiosis, a state characterized by little intracellular water and no metabolic activity. Just to cite some examples, in anhydrobiotic conditions, tardigrades $[1,2]$ produce intracellular trehalose and glycerol, while the nematode Aphelenchus [3] accumulates trehalose during desiccation and glycerol after this phase, and the arctic insect Megaphorura arctica uses trehalose and glycerol to change the membrane composition during dehydration $[4,5]$. The trehalose and glycerol synthesis by these organisms stimulated scientific studies on those systems individually and more recently on their mixtures in order to understand the molecular mechanisms responsible for their bioprotective functions and to open the way for industrial applications of these systems, in particular, for the preservation of protein structure and the maintaining of the biomolecular functions [6-17].

From the physical point of view, the carbohydrate trehalose ( $\alpha$-Dglucopyranosyl $\alpha$-D-glucopyranoside, $\mathrm{C}_{12} \mathrm{H}_{22} \mathrm{O}_{11}$,
$M_{w}=345$ a.u.) and the alcohol glycerol (1,2,3-propanetriol, $\mathrm{C}_{3} \mathrm{O}_{3} \mathrm{H}_{8}, M_{w}=92$ a.u.) are glass-forming systems. Trehalose possesses two crystalline stable phases, one dihydrated and one anhydrous, and has a glass transition temperature $T_{g}$ of $388 \mathrm{~K}$. On the other hand, glycerol shows an intrinsic difficulty to crystallize and has a $T_{g}$ of $190 \mathrm{~K}$. Furthermore, trehalose and glycerol are classified as "intermediate" in the Angell "strong-fragile" scheme [18-21], which ranks systems on the basis of the structure sensitivity to temperature changes in approaching the glass transition, and it has been shown that the effect of trehalose on biomolecular structural and dynamical properties is mediated by water, whose tetrahedral network is strongly deformed by the sugar [2225], while the sugar hydrogen-bonded network is affected by the presence of glycerol, which by means of strong attractive forces creates intense hydrogen-bonded interactions [15-17].

With the aim to investigate the different bioprotective effectiveness of trehalose and trehalose/glycerol mixtures, a systematic study on mixtures at different glycerol concentration values in trehalose $(0.00 \%, 1.25 \%, 2.50 \%, 5.00 \%$, $7.50 \%$, and $10.00 \%$ by weight) has been performed [17-19]. 
The present review article shows an overview of INS findings on trehalose/glycerol mixtures as a function of $Q$, temperature, and concentration. The data analysis is focused on the Boson peak features at different temperatures and then on the fragility behavior in order to relate these properties to the biological role of trehalose and glycerol.

\section{Materials and Methods}

Ultrapure dihydrated trehalose and glycerol, purchased by Aldrich-Chemie, were used for sample preparation. Homogeneous and dust-free samples were stabilized for an ample time $(30 \mathrm{~min})$ to reach the thermal equilibrium before each temperature run. The investigated glycerol concentration values in the trehalose/glycerol mixtures are $0.00 \%, 1.25 \%$, $2.50 \%, 5.00 \%, 7.50 \%$, and $10.00 \%$ by weight.

INS experiments were carried out by using the OSIRIS spectrometer at the ISIS Facility (Rutherford Appleton Laboratory, Oxford, UK) and by using the IN4 and IN6 spectrometers at the Institute Laue Langevin (ILL, Grenoble, France). OSIRIS is an inverted geometry time-of-flight instrument such that neutrons scattered by the sample are energy-analyzed by means of Bragg scattering from largearea crystal-analyzer array. The configuration of OSIRIS used for the INS measurements was scattering angle range of $11^{\circ}<2 \theta<55^{\circ}$, PG004 graphite with a momentum transfer range of $0.7 \AA^{-1}<Q<3.6 \AA^{-1}$, and energy resolution of $99 \mu \mathrm{eV}$ (FWHM). The IN4 spectrometer is a time-of-flight spectrometer used for the study of excitations in condensed matter, and it was configured for the measurements with an incident wavelength of $2.96 \AA$ and an energy resolution of $450 \mu \mathrm{eV}$. The IN6 spectrometer is a time-of-flight spectrometer designed for quasielastic and inelastic scattering for incident wavelengths in the range of 4 to $6 \AA$. The incident wavelength used for the measurements was $5.12 \AA$ with an energy resolution of $50 \mu \mathrm{eV}$.

\section{Results and Discussion}

The Boson peak is still subject of controversy in the physics of glasses [26-30], promoted largely by the recognition that excitations of Boson peak energies are responsible for low temperature anomalies in glasses (e.g., violations of the Debye $T^{3}$ law for low-temperature heat capacity). While clearly identified as an excess in the vibrational density of states over that expected for a homogeneous elastic solid, the Boson peak detailed character has been very difficult to establish [26, 27]. In the opinion of some authors [28], this vibrational bump marks the crossover from propagating sound waves to vibrations localized at the wavelength towards the typical length scales for which the disorder of the material becomes significant. On the other hand, other authors [29] have attributed the Boson peak existence to lowest-order intermolecular vibrations of clusters of atoms or to fractal structures. Finally, the so-called soft potential model [30] attributes this vibrational contribution to the coexistence of soft vibrations of a collection of particles and sound waves for frequencies up to the maximum of the peak.
In the one-phonon approximation [31], the incoherent dynamic structure factor at a given temperature $T$ can be written as

$$
\begin{aligned}
S(Q, \omega)= & \exp \left(-Q^{2}\left\langle u^{2}\right\rangle\right) \\
& \times\left\{\operatorname{EISF}(Q) \delta(\omega)+[1-\operatorname{EISF}(Q)] S_{\mathrm{QE}}(Q, \omega)\right\} \\
& +S_{\operatorname{INEL}}(Q, \omega)
\end{aligned}
$$

where $\exp \left(-Q^{2}\left\langle u^{2}\right\rangle\right)$, with $Q$ the exchanged wavevector and $\left\langle u^{2}\right\rangle$ mean square vibration amplitude, is the Debye-Waller factor, $\operatorname{EISF}(Q)$ is the elastic incoherent scattering function, $\delta(\omega)$ is the Dirac delta function, $S_{\mathrm{QE}}(Q, \omega)$ is the quasielastic incoherent dynamic structure factor, and $S_{\text {INEL }}(Q, \omega)$ is the inelastic incoherent dynamic structure factor. The first term is related to the elastic response of the system, the second term is linked to the diffusive nonvibrational motions and appears as a broadening of the elastic peak, and finally the last term is connected with the vibrational behavior. The inelastic contribution has been observed [32] to become less distinct due to the overlapping with a broad quasielastic component of increasing intensity, which is accompanied by a decrease in the elastic intensity. By assuming that, only incoherent scattering is visible, this latter contribution can be written as

$$
S_{\mathrm{INEL}}(Q, \omega)=e^{-2 W(\mathrm{Q}, T)} \frac{Q^{2}}{8 \pi M} \frac{n(\omega, T)}{\omega} g(\omega),
$$

where $M$ is the mass of the scattering unit, $n(\omega, T)$ is the Bose factor, and $g(\omega)$ is the density of vibrational states. In a real experiment, (1) is understood to be convoluted with the energy resolution function of the instrument. In our case, the spectra obtained at $T=100 \mathrm{~K}$ show no quasielastic broadening of the elastic line.

Figure 1 shows the averaged dynamic structure factor for trehalose/glycerol mixtures, obtained by adding up the spectra at all the investigated $Q$ values, as a function of the energy shift at $T=2 \mathrm{~K}, 100 \mathrm{~K}$, and $300 \mathrm{~K}$. From an inspection of Figure 1, the Boson peak is observed for all the investigated glycerol concentration values at $\sim 7 \mathrm{meV}$ at $T=2 \mathrm{~K}$, at $\sim 5 \mathrm{meV}$ at $T=100 \mathrm{~K}$, and at $\sim 4 \mathrm{meV}$ at $T=300 \mathrm{~K}$.

In order to get dynamical information about the investigated systems, the INS spectra have been studied as a function of $Q$, as shown in Figure 2. The Boson peak intensity as a function of $Q$, as shown in Figure 3, suggests both a quasiharmonic behavior and a high cooperative degree in the fast motions of trehalose/glycerol mixtures [33-35].

To quantitatively evaluate the relative importance of the relaxational versus vibrational contributions, we also apply the procedure introduced by Sokolov et al. for determining the fragility of glass-forming systems [36, 37]. Sokolov and coworkers correlate the ratio of the fast relaxation (anharmonic part) to the vibrational (harmonic part) contributions 


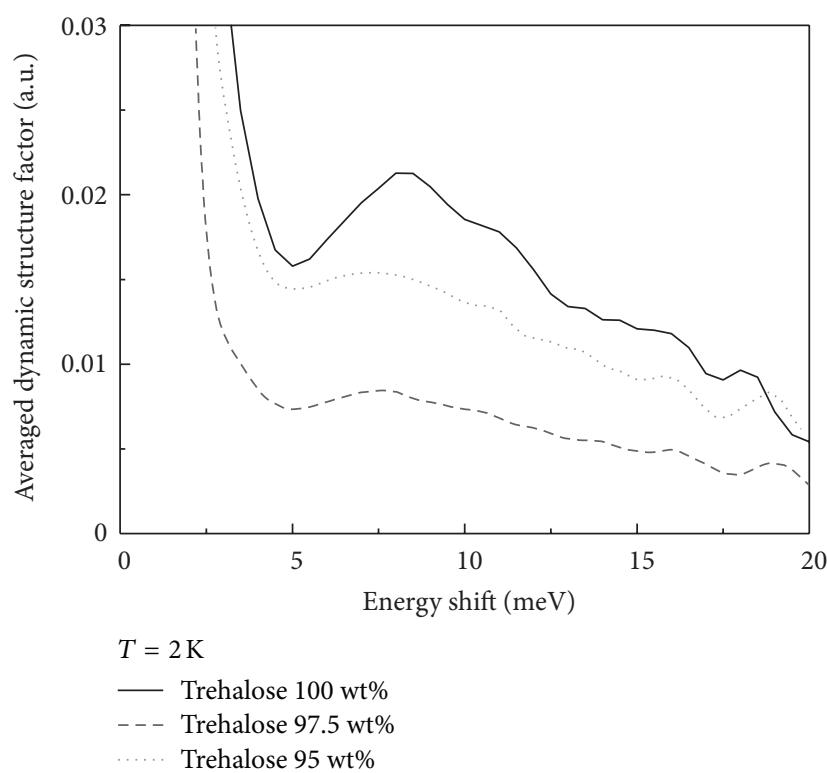

(a)

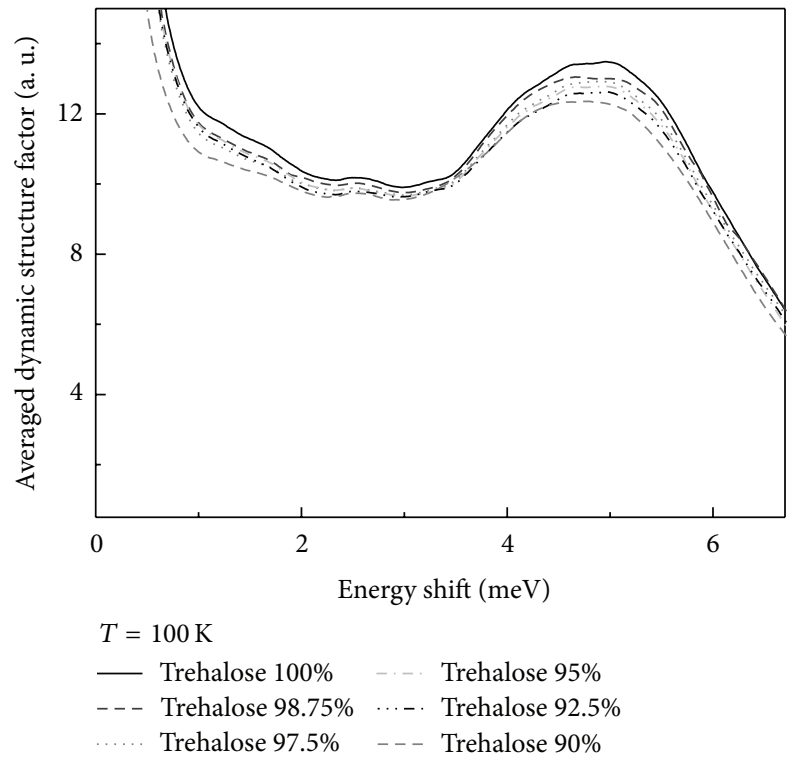

(b)

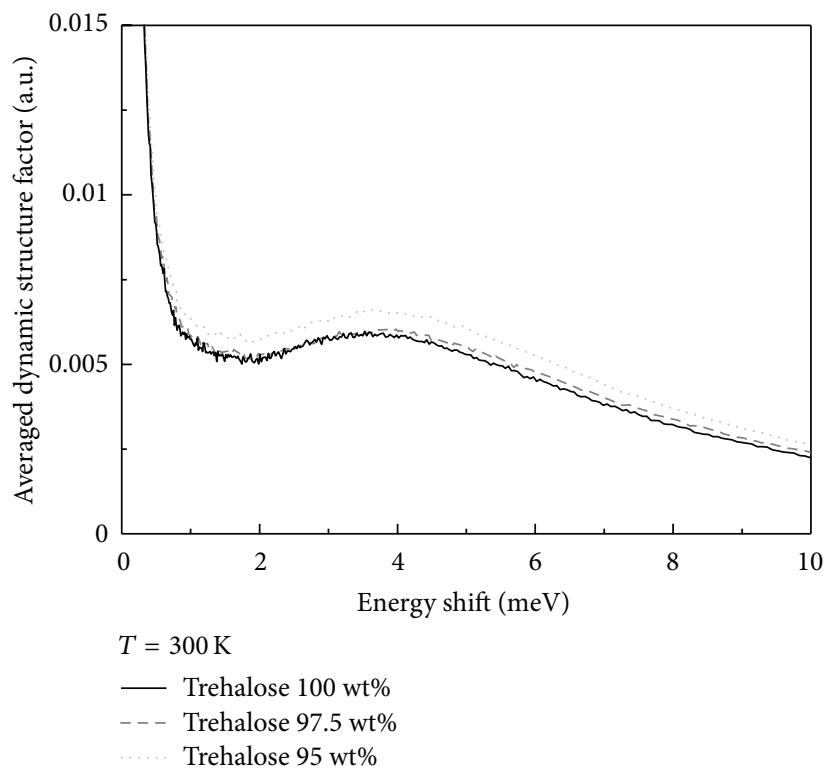

(c)

Figure 1: Averaged dynamic structure factor for trehalose/glycerol mixtures, obtained by adding up the spectra at different $Q$, as a function of the energy shift at (a) $T=2 \mathrm{~K}$ by IN4, (b) $T=100 \mathrm{~K}$ by OSIRIS, and (c) $T=300 \mathrm{~K}$ by IN6.

to the fast dynamics at $T_{g}$ with the degree of fragility $F$ defined as

$$
F=\frac{E_{\eta}}{T_{g}}=\frac{R}{T_{g}}\left(\frac{\partial \ln \eta}{\partial(1 / T)}\right)_{T=T_{g}}
$$

where $E_{\eta}$ is the apparent activation energy of viscous flow at $T_{g}$ normalized by $T_{g}, R$ is the gas constant, and $\eta$ represents the shear viscosity. Experimentally, the magnitude of the Boson peak is found to be greater for strong glasses as silica and to decline in strength as fragility increases. However, when the density of silica is reduced, the intensity of the
Boson peak increases further, suggesting that the strength of the glass may also be increasing [38].

Following Sokolov et al. [36, 37], the ratio of anharmonic to harmonic contribution can be measured as a ratio $R_{1}$ of the normalized intensity at the minimum $I_{\min }$ to the normalized intensity at the maximum $I_{\max }$ as follows:

$$
R_{1}=\frac{I_{\min }}{I_{\max }} .
$$

In this framework, since $R_{1}$ increases monotonically with increasing degree of fragility, a higher value of $R_{1}$ can be attributed to a more fragile character $[36,37]$. 


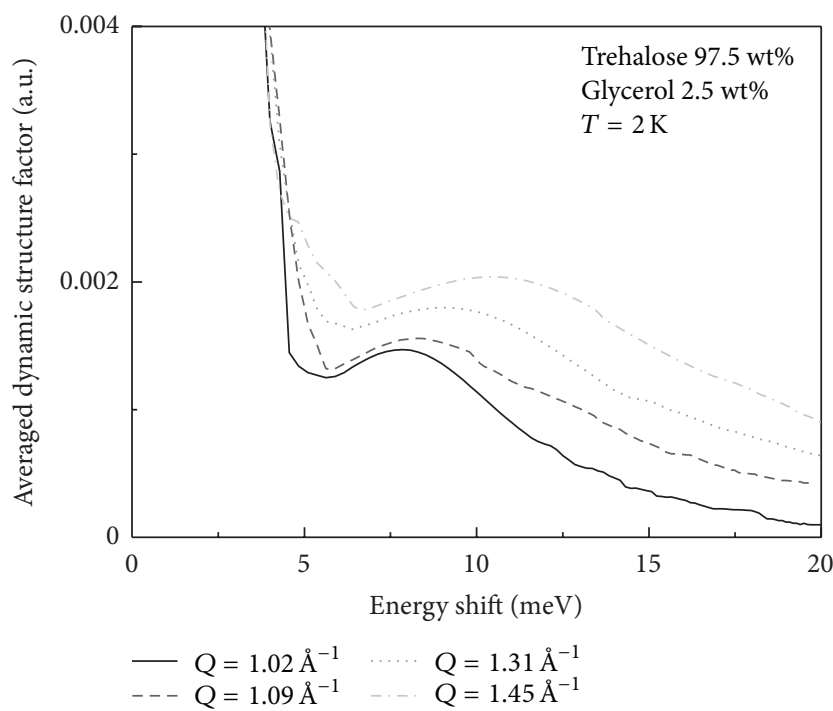

(a)

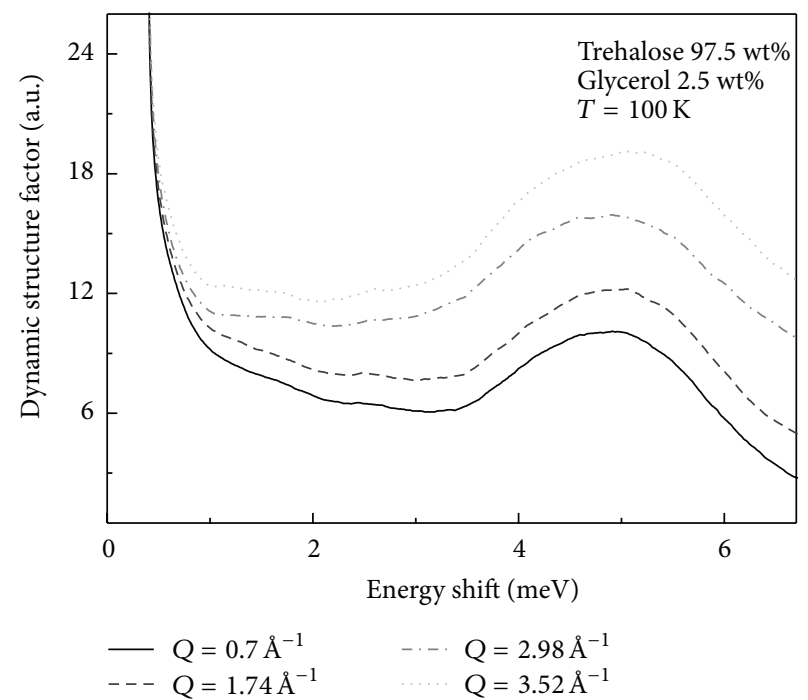

(b)

Figure 2: Dynamic structure factor for a trehalose/glycerol mixture at $2.5 \mathrm{wt} \%$ of glycerol as a function of the energy shift for different $Q$ values at (a) $T=2 \mathrm{~K}$ and (b) $T=100 \mathrm{~K}$.

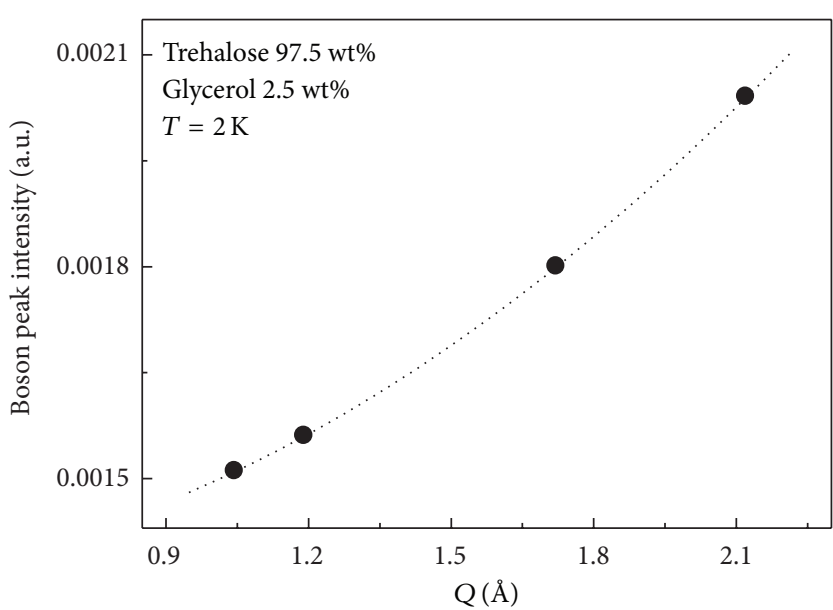

(a)

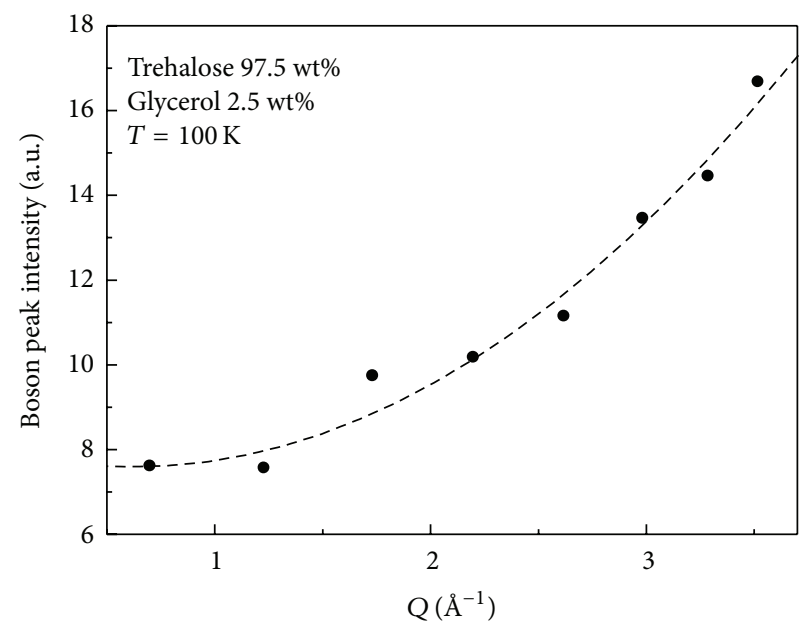

(b)

Figure 3: $Q$ dependence of the Boson peak intensity for a trehalose/glycerol mixture at $2.5 \mathrm{wt} \%$ of glycerol as a function of the energy shift for different $Q$ values at (a) $T=2 \mathrm{~K}$ and (b) $T=100 \mathrm{~K}$.

The $R_{1}$ parameter behavior as a function of glycerol concentration at $T=2 \mathrm{~K}, 100 \mathrm{~K}$, and $300 \mathrm{~K}$ is shown in Figure 4. It is highlighted that the addition of small amounts of glycerol to trehalose significantly changes its fragility. Beyond this point, fragility first decreases and then increases with the further addition of glycerol. Clearly, glycerol affects the fragility of sugar in a nonmonotonic manner, showing a minimum at the glycerol concentration value of $2.5 \%$.

It has been shown $[12-17,39,40]$ that the addition of small amounts of a low $T_{g}$ diluent to pure trehalose leads to a reduced $T_{g}$ and an increased stability for proteins sequestered in the glass. Specifically, the stability of enzymes embedded in mixtures of glycerol and trehalose with various compositions has been probed by measuring the deactivation times of the enzymes. In particular, Cicerone and coworkers showed that the addition of $5 \%$ by weight of glycerol to pure trehalose gives rise to the smallest mean square displacement. Furthermore, these authors confirmed the correlation between the preservation of enzymes and the suppression of short time local dynamics [15].

The INS findings suggest, therefore, that on a short wavelength and time scale, the local fast dynamics, which can be linked to the protein stabilization effectiveness, is in a more marked way suppressed for a glycerol concentration value of $2.5 \%$. On the other hand, this stronger suppression of the fast dynamics can be correlated with a less fragile character. 


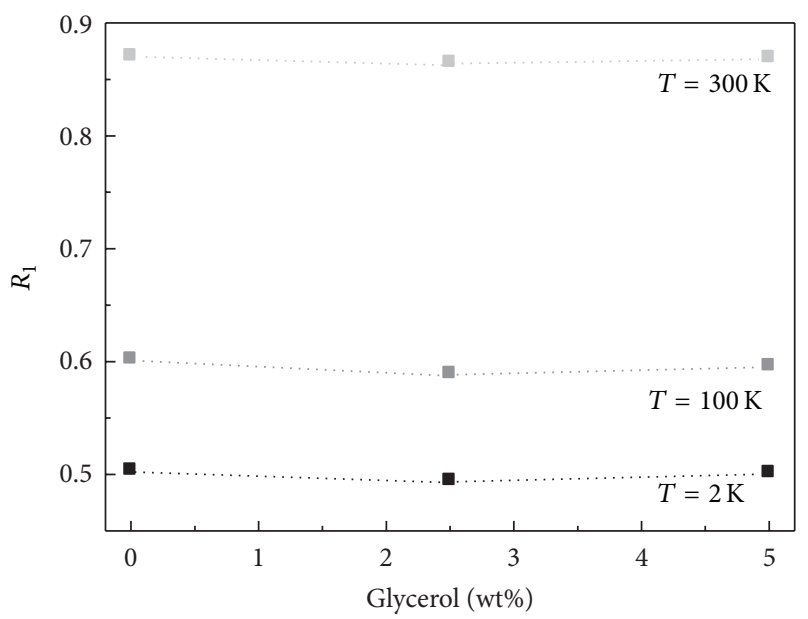

FIGURE 4: $R_{1}$ parameter behaviour as a function of glycerol concentration for trehalose/glycerol mixtures at $T=2 \mathrm{~K}$ by IN4, $T=100 \mathrm{~K}$ by OSIRIS, and $T=300 \mathrm{~K}$ by IN6.

\section{Conclusions}

In order to get information about the effect of the presence of glycerol on trehalose, the Boson peak behavior as a function of $Q$ and concentration in trehalose/glycerol mixtures has been analyzed. The obtained information allowed determining the fragility degree and its dependence on glycerol content.

The whole body of data on trehalose/glycerol mixtures at different glycerol content supports the hypothesis that, in the small investigated glycerol concentration range, the trehalose/glycerol matrix forms a stronger hydrogen-bonding network with respect to that of pure trehalose. The signature of the strengthening of the hydrogen-bonded network created by trehalose and glycerol is recognizable in the trends followed by all the determined physical quantities. More specifically, the increased rigidity revealed by the dynamic features confirms that the hydrogen-bonded interactions are rearranged in a stronger network as a consequence of the addition of glycerol. The molecular origin of this anomalous behavior can be linked to the registered minima in the $R_{1}$ parameter, which clearly signals the presence of a not-ideal mixing process.

The present INS findings, which have been obtained from very low to very high temperature, can also provide a physical explanation for the trehalose-glycerol interplay found in several organisms under stress conditions as well as they can furnish some useful details about the biotechnological use of trehalose and glycerol.

\section{References}

[1] S. Hengherr, F. Brünner, and R. O. Schill J, "Anhydrobiosis in tardigrades and its effects on longevity traits," Journal of Zoology, vol. 275, no. 3, pp. 216-220, 2008.

[2] R. O. Schill and G. B. Fritz, "Desiccation tolerance in embryonic stages of the tardigrade," Journal of Zoology, vol. 276, no. 1, pp. 103-107, 2008.
[3] J. A. Browne, K. M. Dolan, T. Tyson, K. Goyal, A. Tunnacliffe, and A. M. Burnell, "Dehydration-specific induction of hydrophilic protein genes in the anhydrobiotic nematode Aphelenchus avenae," Eukaryotic Cell, vol. 3, no. 4, pp. 966-975, 2004.

[4] X. He, A. Fowler, and M. Toner, "Water activity and mobility in solutions of glycerol and small molecular weight sugars: implication for cryo- and lypopreservation," Journal of Applied Physics, vol. 100, Article ID 074702, 2006.

[5] M. R. Michaud and D. L. Denlinger, "Shifts in the carbohydrate, polyol, and amino acid pools during rapid cold-hardening and diapause-associated cold-hardening in flesh flies (Sarcophaga crassipalpis): a metabolomic comparison," Journal of Comparative Physiology B, vol. 177, pp. 753-763, 2007.

[6] S. E. Pagnotta, M. A. Ricci, F. Bruni, S. McLain, and S. Magazù, "Water structure around trehalose," Chemical Physics, vol. 345, no. 2-3, pp. 159-163, 2008.

[7] G. Caliskan, D. Mechtani, S. Azzam et al., "Protein and solvent dynamics: how strongly are they coupled?" Journal of Chemical Physics, vol. 121, no. 4, pp. 1978-1983, 2004.

[8] J.-A. Seo, S. J. Kim, H.-J. Kwon, Y. S. Yang, H. K. Kim, and Y.-H. Hwang, "The glass transition temperatures of sugar mixtures," Carbohydrate Research, vol. 341, no. 15, pp. 2516-2520, 2006.

[9] B. Varga, F. Migliardo, E. Takacs, B. Vertessy, S. Magazù, and C. Mondelli, "Neutron scattering studies on dUTPase complex in the presence of bioprotectant systems," Chemical Physics, vol. 345, no. 2-3, pp. 250-258, 2008.

[10] B. Varga, F. Migliardo, E. Takacs, B. Vertessy, S. Magazù, and M. T. F. Telling, "Study of solvent-protein coupling effects by neutron scattering," Journal of Biological Physics, vol. 36, no. 2, pp. 207-220, 2010.

[11] S. Magazù, F. Migliardo, A. Benedetto, and B. Vertessy, "Protein dynamics by neutron scattering: the protein dynamical transition and the fragile-to-strong dynamical crossover in hydrated lysozyme," Chemical Physics, 2013.

[12] S. Magazù, F. Migliardo, F. Affouard, M. Descamps, and M. T. F. Telling, "Study of the relaxational and vibrational dynamics of bioprotectant glass-forming mixtures by neutron scattering and molecular dynamics simulation," Journal of Chemical Physics, vol. 132, no. 18, Article ID 184512, 9 pages, 2010.

[13] S. Magazù, F. Migliardo, and M. T. F. Telling, "Dynamics of glass-forming bioprotectant systems," Journal of NonCrystalline Solids, vol. 357, no. 2, pp. 691-694, 2011.

[14] S. Magazù, F. Migliardo, M. A. Gonzalez, and C. Mondelli, "Inelastic neutron scattering study of dynamical properties of bioprotectant solutions against temperature," Journal of NonCrystalline Solids, vol. 358, no. 18-19, pp. 2635-2640, 2012.

[15] M. T. Cicerone and C. L. Soles, "Fast dynamics and stabilization of proteins: binary glasses of trehalose and glycerol," Biophysical Journal, vol. 86, no. 6, pp. 3836-3845, 2004.

[16] J. E. Curtis, T. E. Dirama, G. A. Carri, and D. J. Tobias, "Inertial suppression of protein dynamics in a binary glycerol-trehalose glass," Journal of Physical Chemistry B, vol. 110, no. 46, pp. 22953-22956, 2006.

[17] S. Magazù, F. Migliardo, and S. F. Parker, "Vibrational properties of bioprotectant mixtures of trehalose and glycerol," The Journal of Physical Chemistry B, vol. 115, no. 37, pp. 11004-11009, 2011.

[18] F. Affouard, P. Bordat, M. Descamps et al., "A combined neutron scattering and simulation study on bioprotectant systems," Chemical Physics, vol. 317, no. 2-3, pp. 258-266, 2005. 
[19] S. Magazù, G. Maisano, and F. Migliardo, "Fragility by elastic incoherent neutron scattering," Journal of Chemical Physics, vol. 121, no. 18, pp. 8911-8915, 2004.

[20] I. V. Blazhnov, S. Magazù, G. Maisano, N. P. Malomuzh, and F. Migliardo, "Macro- and microdefinitions of fragility of hydrogen-bonded glass-forming liquids," Physical Review E, vol. 73, no. 3, Article ID 031201, 2006.

[21] S. Magazù, F. Migliardo, C. Mondelli, and M. Vadalà, "Correlation between bioprotective effectiveness and dynamic properties of trehalose-water, maltose-water and sucrose-water mixtures," Carbohydrate Research, vol. 340, no. 18, pp. 2796-2801, 2005.

[22] S. Magazu, F. Migliardo, and M. T. F. Telling, "Study of the dynamical properties of water in disaccharide solutions," European Biophysics Journal, vol. 36, no. 2, pp. 163-171, 2007.

[23] S. Magazù, F. Migliardo, and M. T. F. Telling, "Structural and dynamical properties of water in sugar mixtures," Food Chemistry, vol. 106, no. 4, pp. 1460-1466, 2008.

[24] S. Magazù, F. Migliardo, C. Mondelli, and G. Romeo, “Temperature dependence of mean square displacement by IN13: a comparison between trehalose and sucrose water mixtures," Chemical Physics, vol. 292, no. 2-3, pp. 247-251, 2003.

[25] S. Magazù, G. Maisano, F. Migliardo, and C. Mondelli, “ $\alpha, \alpha-$ trehalose/water solutions. VII: an elastic incoherent neutron scattering study on fragility," The Journal of Physical Chemistry $B$, vol. 108, no. 36, pp. 13580-13585, 2004.

[26] M. Neumann, "The dielectric constant of water. Computer simulations with the MCY potential ", Journal of Chemical Physics, vol. 82, no. 12, Article ID 5663, 10 pages, 1985.

[27] H. J. C. Berendsen, J. P. M. Postma, W. F. Van Gunsteren, A. Dinola, and J. R. Haak, "Molecular dynamics with coupling to an external bath," The Journal of Chemical Physics, vol. 81, no. 8, pp. 3684-3690, 1984.

[28] S. A. Elliott, "A unified model for the low-energy vibrational behaviour of amorphous solids," Europhysics Letters, vol. 19, no. 3, article 201, 1992.

[29] R. L. Orbach, T. Nakayama, and K. Yakubo, "Dynamical properties of fractal networks: scaling, numerical simulations, and physical realizations," Reviews of Modern Physics, vol. 66, no. 2, pp. 381-443, 1994.

[30] V. G. Karpov, Y. M. Galperin, and V. I. Kozub, "Localized states in glasses," Advances in Physics, vol. 38, no. 6, pp. 669-737, 1989.

[31] M. Bée, Quasielastic Neutron Scattering: Principles and Applications in Solid-State Chemistry, Biology and Material Science, Hilger, Bristol, 1988.

[32] A. M. Gaspar, W. Doster, R. Gebhardt, and T. Unruh, "Pressureinduced dissociation of casein micelles: size distribution and effect of temperature," Brazilian Journal of Medical and Biological Research, vol. 38, no. 8, pp. 1209-1214, 2005.

[33] H. Shintani and H. Tanaka, "Universal link between the boson peak and transverse phonons in glass," Nature Materials, vol. 7, no. 11, pp. 870-877, 2008.

[34] D. Fioretto, U. Buchenau, L. Comez et al., "High-frequency dynamics of glass-forming polybutadiene," Physical Review E, vol. 59, no. 4, pp. 4470-4475, 1999.

[35] H. Nakagawa, M. Kataoka, Y. Joti et al., "Hydration-coupled protein boson peak measured by incoherent neutron scattering," Physica B, vol. 385-386, pp. 871-873, 2006.

[36] A. P. Sokolov, A. Kisliuk, D. Quitmann, and E. Duval, "Evaluation of density of vibrational states of glasses from lowfrequency Raman spectra," Physical Review B, vol. 48, no. 10, pp. 7692-7695, 1993.
[37] A. P. Sokolov, E. Rossler, A. Kisliuk, and D. Quitmann, "Dynamics of strong and fragile glass formers: differences and correlation with low-temperature properties," Physical Review Letters, vol. 71, no. 13, pp. 2062-2065, 1993.

[38] C. N. Greaves, F. Meneau, O. Majérus, D. G. Jones, and J. Taylor, "Chemistry: identifying vibrations that destabilize crystals and characterize the glassy state," Science, vol. 308, no. 5726, pp. 1299-1302, 2005.

[39] P. Bordat, A. Lerbret, J.-P. Demaret, F. Affouard, and M. Descamps, "Comparative study of trehalose, sucrose and maltose in water solutions by molecular modelling," Europhysics Letters, vol. 65, no. 1, pp. 41-47, 2004.

[40] T. E. Dirama, G. A. Carri, and A. P. Sokolov, "Role of hydrogen bonds in the fast dynamics of binary glasses of trehalose and glycerol: a molecular dynamics simulation study," The Journal of Chemical Physics, vol. 122, no. 11, Article ID 114505, 2005. 

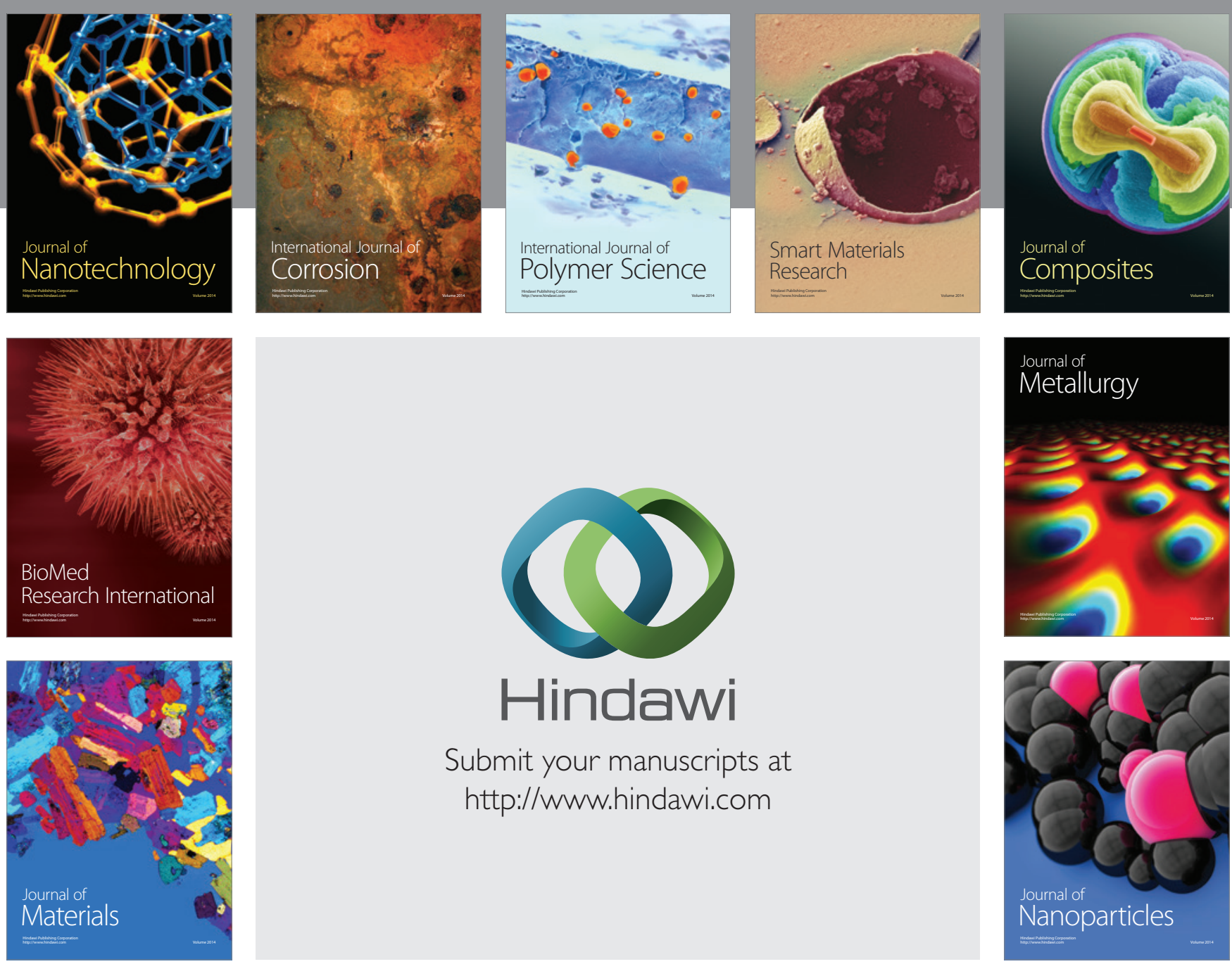

Submit your manuscripts at http://www.hindawi.com
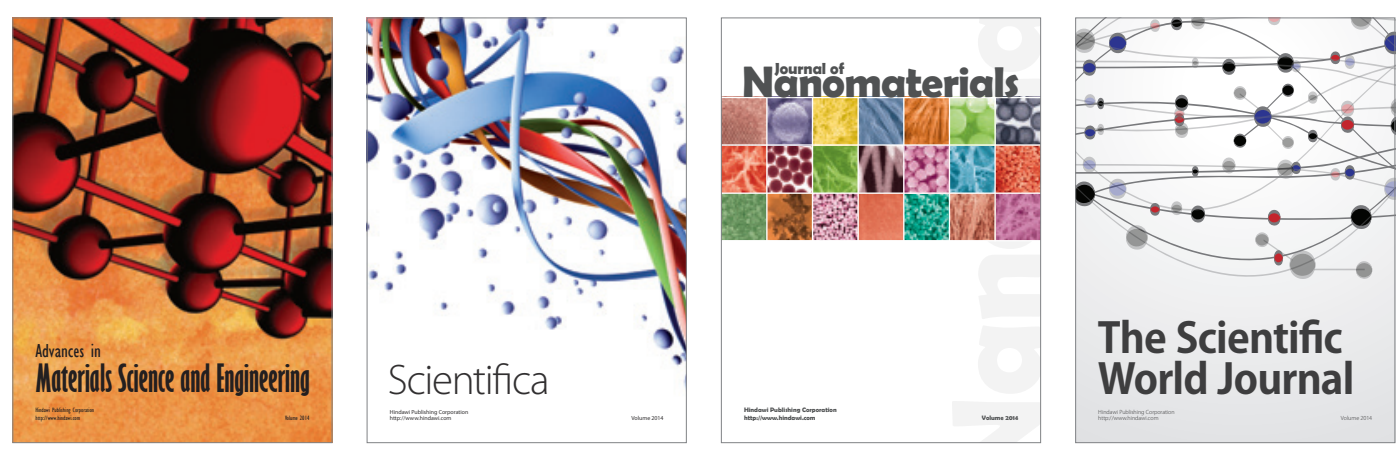

\section{The Scientific World Journal}
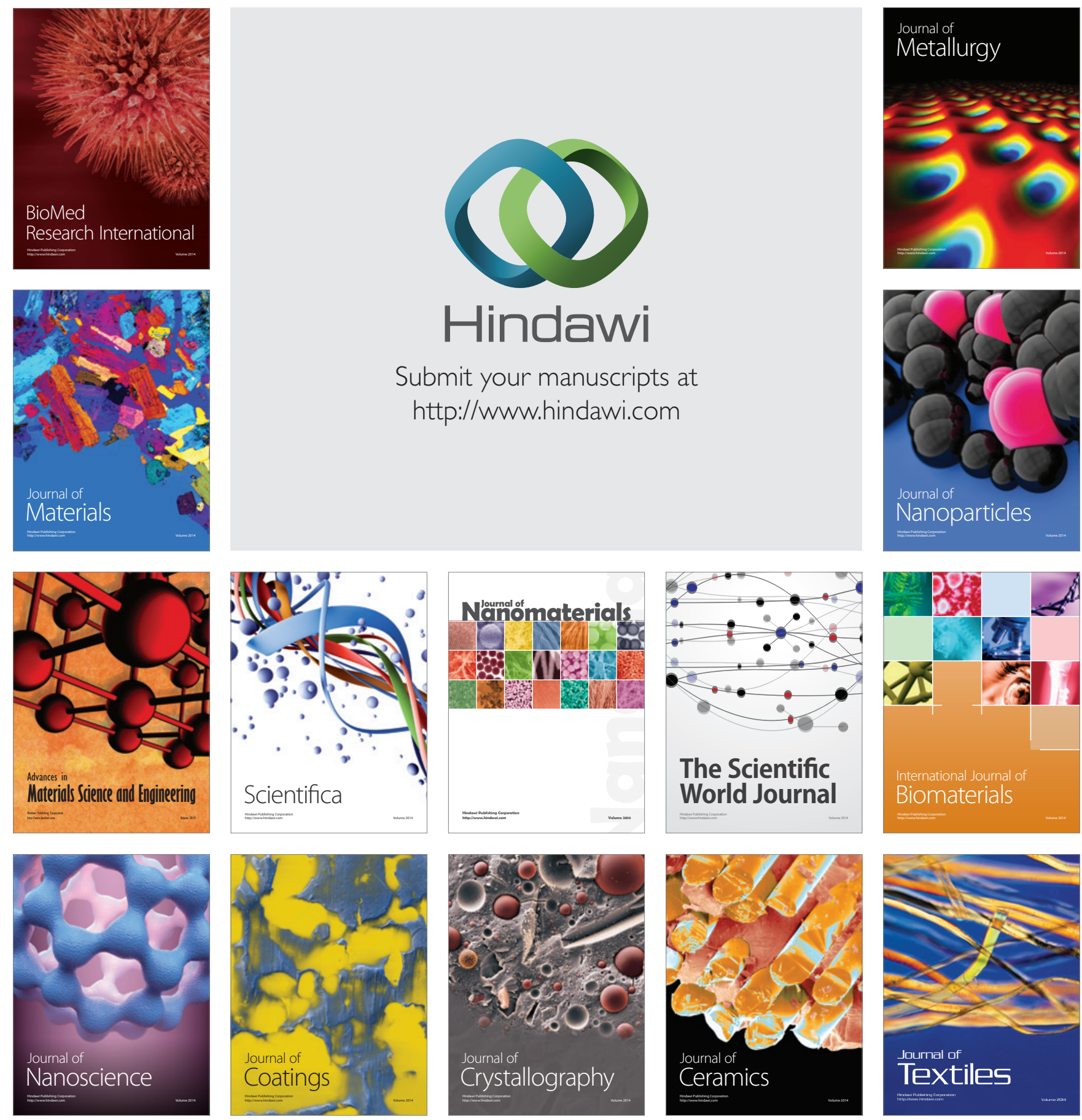